INPLASY PROTOCOL

To cite: Cheng et al. Efficacy and safety of the apolipoprotein C3 inhibitor Volanesorsen in familial hypertriglyceridemia syndrome: a systematic review and meta-analysis. Inplasy protocol 2021120111. doi: 10.37766/inplasy2021.12.0111

Received: 24 December 2021

Published: 24 December 2021

Corresponding author: Yonglang Cheng

chengyonglang666@163.com

Author Affiliation:

Affiliated Hospital of

Southwest Medical University.

Support: No. 82170587.

Review Stage at time of this submission: Data extraction Completed but not published.

Conflicts of interest:

None declared.

\section{Efficacy and safety of the apolipoprotein C3 inhibitor Volanesorsen in familial hypertriglyceridemia syndrome: a systematic review and meta-analysis}

Cheng, Y1; Li, T2; Tan, P3; Du, Y4; Huang, Z5; Shi, H6; Cai, T7; Chen, Y8; Fu, W9.

Review question / Objective: Familial hypertriglyceridemia syndrome (FCS) is a hypertriglyceridemia (HTG) caused by the accumulation of celiac particles. Recently, an increasing number of studies have shown that Volanesorsen can improve lipid metabolism and be used in the treatment of patients with FCS and HTG. To evaluate the effect of Volanesorsen on lipid metabolism in patients with FCS and to investigate whether it causes more adverse events, we performed a systematic evaluation and meta-analysis.

Condition being studied: Efficacy and safety of the apolipoprotein C3 inhibitor Volanesorsen in familial hypertriglyceridemia syndrome.

INPLASY registration number: This protocol was registered with the International Platform of Registered Systematic Review and Meta-Analysis Protocols (INPLASY) on 24 December 2021 and was last updated on 24 December 2021 (registration number INPLASY2021120111).

\section{INTRODUCTION}

Review question / Objective: Familial hypertriglyceridemia syndrome (FCS) is a hypertriglyceridemia (HTG) caused by the accumulation of celiac particles. Recently, an increasing number of studies have shown that Volanesorsen can improve lipid metabolism and be used in the treatment of patients with FCS and HTG. To evaluate the effect of Volanesorsen on lipid metabolism in patients with FCS and to investigate whether it causes more adverse events, we 
performed a systematic evaluation and meta-analysis.

Rationale: The meta-analysis followed the Preferred Reporting Items for Systematic Reviews and Meta-Analyses (PRISMA) guidelines.Randomized controlled trials published between January 1, 2000 and November 1, 2021 were provided by PubMed, Embase, ClinicalTrials.gov and the Cochrane Library. This study included all randomized controlled trials without language restrictions.

Condition being studied: Efficacy and safety of the apolipoprotein C3 inhibitor Volanesorsen in familial hypertriglyceridemia syndrome.

\section{METHODS}

Search strategy: The literature search was conducted using Cochrane Library, the PubMed database, the Embase database, the ClinicalTrials.gov, and the bibliography of the original articles was manually checked. The medical subject heading keywords used including "Volanesorsen", "ISIS 304801", "Hyperlipoproteinemia Type I","Familial hyperchylomicronemia".was used to search for Volanesorsen and familial hyperceliac syndrome. The reference lists of retrieved studies were manually checked to identify further relevant studies.

Participant or population: The selected study population included HTG patients, FCS patients,FPL Patients and healthy adults.

Intervention: Volanesorsen.

Comparator: Placebo.

Study designs to be included: Randomized placebo-controlled clinical trials

Eligibility criteria: Studies included in this meta-analysis met the following criteria. (1) randomized controlled trials (RCTs) or clinical trials; (2) interventions using Volanesorsen and where the control group was placebo or no treatment. and (3) studies involving measures of lipid metabolism such as triglycerides (TG), total cholesterol (TC), high-density lipoprotein (HDL) and low-density lipoprotein (LDL), Body-mass index (BMI), High-density lipoprotein cholesterol (HDL-C), Low density lipoprotein cholesterol (LDL-C), apo C-III, etc. (4) The patient's BMI $\leq 45 \mathrm{~kg} / \mathrm{m} 2$, Fasting TG $\geq 500 \mathrm{mg} / \mathrm{dL}$ ( $\geq 5.7 \mathrm{mmol} / \mathrm{L}$ ) at Screening. The available data were used to calculate the corresponding statistics. Animal studies, review articles and conference abstracts were not included.

Information sources: The literature search was conducted using Cochrane Library, the PubMed database, the Embase database, and the ClinicalTrials.gov .

Main outcome(s): Four randomized, controlled trials involving $\mathbf{2 4 6}$ patients were analyzed for this study. Patients treated with Volanesorsen showed (MD = -78.85\%; $95 \% \mathrm{Cl}=-96.04$ to $-61.65, \mathrm{p}=0.67, \mathrm{I2}=0 \%$ ) decrease in TG and (MD = -80.08\% ;95\% $\mathrm{Cl}=-90.02$ to $-71.54, \mathrm{p}=0.25, \quad \mathrm{I}=29 \%$ ) decrease in apo C-III compared to patients in the placebo group showing a significant decrease. In addition, HDL-C increased (MD $=46.01 \% \quad 95 \% \quad C I=41.03$ to $50.99, P=0.41, \quad 12=0 \%)$, NHDL-C decreased $(M D=-32.12 \% ; 95 \% C l=-44.39$ to $-19.85, P=0.11, I 2=55 \%)$, VLDL-C decreased $(M D=-65.88 \% ; 95 \% \mathrm{Cl}=-83.97$ to $-47.79, \mathrm{P}=0.71, \quad \mathrm{I}=0 \%)$, apo $\mathrm{A1}$ increased $(M D=13.12 \% ; 95 \% \mathrm{CI}=7.83$ to $18.40, P=0.72, I 2=0 \%)$, and apo $B$ increased (MD = $7.94 \% ; 95 \% \mathrm{Cl}=-1.90$ to 17.78, $\mathrm{P}=0.54$, $12=0 \%$ ) all suggest that Volanesorsen has an overall FCS with a therapeutic effect. However, LDL-C increased (MD $=99.59 \%$; $95 \% \mathrm{Cl}=69.19$ to $130.00, \mathrm{P}=0.61, \mathrm{I}=0 \%$ ) and apo B48 decreased (MD $=82.89 \%$; 95\% $\mathrm{Cl}=-100.88$ to $-64.91, \mathrm{P}=0.42, \quad \mathrm{I}=0 \%$ ), showing an inverse effect, suggesting that Volanesorsen's did not target all proteins of lipid metabolism.

Additional outcome(s): In summary, Volanesorsen, as an approved drug for FCS that has passed Phase I clinical trials, can improve lipid metabolism in FCS patients overall and reduce $\mathrm{TG}$, apo $\mathrm{C}$-III. The results of HDL-C, VLDL-C, NHDL-C, apo 
A1, and apo B support these conclusions. However, it is noteworthy that the elevation of LDL-C and the decrease of apo B48 do not support the above results, which may suggest that Volanesorsen does not show positive effects against all lipid metabolism proteins. In addition, Volanesorsen dosing was seen to have a significant increase in Injection site-related adverse events compared to the placebo group in terms of adverse event performance, which may become a disadvantage and needs to be further addressed.

Data management: The statistical analyses were conducted using RevMan software (version 5.3, Cochrane, Oxford, UK). Findings were quantitatively assessed using a combination of fixed effects models or random effects models, based on the level of between-study heterogeneity, using the Higgins index (12). Outcome indicators of lipid changes were expressed as mean difference percentage (MD) and $95 \% \mathrm{Cl}$. Safety analysis was performed using the Mantel-Haenszel method to calculate odd ratios (OR) and $95 \% \mathrm{Cl}$ intervals.

Quality assessment / Risk of bias analysis: Study quality was evaluated using the randomized controlled trials bias tool in the Cochrane Handbook of Evaluation. Six dimensional parameters were assessed, random sequence generation (selection bias), allocation concealment (selection bias), blinding of outcome assessment (detection bias), incomplete outcome data (attrition bias), selective reporting (reporting bias), and other biases. Each parameter was categorized as low risk of bias (+), high risk of bias (-), or unclear ( \pm ).

Strategy of data synthesis: The statistical heterogeneity between studies was evaluated with 12 statistics. The 12 values of $50 \%$, and $75 \%$ as cut-off points represented low, moderate, and high degrees of heterogeneity, respectively, and when the 12 value was greater than $60 \%$, it was a high degree of heterogeneity; then, subgroup analyses were performed. Sensitivity analysis was conducted by eliminating each study in turn. Data were combined using a random-effects model.
All tests were two-tailed, and $p<0.05$ was considered statistically significant.

Subgroup analysis: No subgroup analysis was performed.

Sensitivity analysis: Sensitivity analysis was not performed.

Language: This study included all randomized controlled trials without language restrictions.

Country(ies) involved: China.

Keywords: Volanesorsen; familial hypercoeliac disease syndrome; lipid metabolism; systematic evaluation; Metaanalysis.

Contributions of each author:

Author 1 - Yonglang Cheng - Retrieving literature, analyzing data, drafting manuscripts.

Email: chengyonglang666@163.com

Author 2 - Tongxi Li - Retrieving literature, analyzing data, drafting manuscripts.

Email: Itx5662@163.com

Author 3 - Peng Tan - analyzing data.

Author 4 - Yichao Du - analyzing data.

Author 5 - Zhiwei Huang - Retrieving literature.

Author 6 - Hao Shi - Retrieving literature.

Author 7 - Tianying Cai - Data analysis and verification.

Author 8 - Yifan Chen - Data analysis and verification.

Author 9 - Wenguang Fu - Identify research questions, oversee the research process, and review manuscripts.

Email: fuwg@swmu.edu.cn 